\title{
On capacity expansion planning under strategic and operational uncertainties based on stochastic dominance risk averse management
}

\author{
Laureano F. Escudero' ${ }^{1}$ (D) Juan F. Monge ${ }^{2}$
}

Received: 27 September 2017 / Accepted: 22 May 2018

(C) Springer-Verlag GmbH Germany, part of Springer Nature 2018

\begin{abstract}
A new scheme for dealing with uncertainty in scenario trees is presented for dynamic mixed 0-1 optimization problems with strategic and operational stochastic parameters. Let us generically name this type of problems as capacity expansion planning (CEP) in a given system, e.g., supply chain, production, rapid transit network, energy generation and transmission network, etc. The strategic scenario tree is usually a multistage one, and the replicas of the strategic nodes root structures in the form of either a special scenario graph or a two-stage scenario tree, depending on the type of operational activity in the system. Those operational scenario structures impact in the constraints of the model and, thus, in the decomposition methodology for solving usually large-scale problems. This work presents the modeling framework for some of the risk neutral and risk averse measures to consider for CEP problem solving. Two types of risk averse measures are considered. The first one is a time-inconsistent mixture of the chance-constrained and second-order stochastic dominance (SSD) functionals of the value of a given set of functions up to the strategic nodes in selected stages along the time horizon, The second type is a strategic node-based time-consistent SSD functional for the set of operational scenarios in the strategic nodes at selected stages. A specialization of the nested stochastic decomposition methodology for that problem solving is outlined. Its advantages and drawbacks as well as the framework for some schemes to, at least, partially avoid those drawbacks are also presented.
\end{abstract}

$凶 \quad$ Laureano F. Escudero

laureano.escudero@urjc.es

1 Estadística e Investigación Operativa, Universidad Rey Juan Carlos, URJC, Móstoles, Madrid, Spain

2 Centro de Investigación Operativa, Universidad Miguel Hernández, UMH, Elche, Alicante, Spain 
Keywords Capacity expansion planning - Strategic and tactical uncertainties . Multistage stochastic strategic scenario tree - Two-stage stochastic operational multiperiod scenario tree - Time-consistent and time-inconsistent stochastic dominance

\section{Introduction and motivation}

The realization of the uncertain parameters in mathematical optimization is usually structured in a finite set of scenarios along the periods (in our case, grouped in stages of non-necessarily equal length) in the time horizon. The representation of the uncertain data affects the type of decision to be made in the problem as well as the related model and problem solving decomposition methodologies to be dealt with. So, the quality of the solution offered to the decision making process is also affected by the type of scenario tree to generate in stochastic optimization. While dealing with problems with a large time horizon, say, capacity expansion planing (CEP) and others, there are, undoubtedly, two types of data, two types of uncertainties and two types of variables, namely, strategic and operational ones. The strategic variables are related to the decisions on the location, capacity and timing of the decisions on the infrastructure elements of the CEP of a system, say supply chain, production system, rapid transit network, energy transmission network and energy generation mix system, to name just a few. The operational variables are related to the decisions on the operation planning of the available elements in the system at the periods along the time horizon. So, we have two types of dynamic optimization models, namely, the strategic one and the operational submodels, very different in all aspects and intrinsically inter-related in a usually large-sized global model for real-life problem solving.

The main contributions that we present in this work for dealing with CEP problems are as follows:

- A framework for representing the uncertainty in a multistage non-symmetric scenario tree with strategic nodes in the first period of each stage. For each strategic node, there is either an operational multiperiod scenario special graph or an operational scenario two-stage tree, both in the stages along the time horizon. Each structure is locally related to a strategic node, let its nodes be named operational ones. The graph structure is rooted with as many operational replicas of the related strategic node as the number of operational scenarios that are considered for the stage the strategic node belongs to. It is worth to pointing out that each operational node in the graph is linked to a period in the stage. On the other hand, the tree structure is rooted with just one operational replica of the strategic node, and the operational second stage nodes are the same operational scenarios. Notice that they are only related to the so-named 'reference' period in the stages along the time horizon.

- The investment on the capacity expansion of the system is assumed to be made at the strategic nodes, so that the operation of the available elements of the system is made at the operational nodes. The goal in the proposed model consists of minimizing the net present value (NPV) of the expected investment costs plus operational costs. The strategic and operational submodels are run interconnected 
by the so-called linking variables. On the other hand, there are frequently several functions to be optimized in this type of problems, basically, due to environmental and cultural reasons, besides the usual cost minimization.

- A multi-function risk averse measure is considered in the model for reducing the risk of a negative impact of the proposed solution in low-probability high-cost scenarios (i.e., the so-called black swan scenarios). The measure that is proposed is based on stochastic dominance (SD) functional in the two following flavors. The first one is a second-order SD functional, where the risk averse measure on the values of the function to consider is individualized for the set of operational scenarios in the graphs and trees structures. Those ones are rooted with the related replicas of the strategic nodes in the stages. It is a strategic node-based timeconsistent measure. The second flavor is a mixture of the chance-constrained and second-order SD functionals. Here, the risk reduction in the value of the functions is considered up to the stage where the strategic node with one-to-one correspondence with the concerned strategic scenarios belongs to. Note: The terms of the functions also include those related to the operational scenarios in the strategic nodes. It is a time-inconsistent measure.

It is worth to point out that the time-consistent measure performs the risk management on the operational scenarios in the strategic nodes. The time-inconsistent measure performs the risk management on the global set of strategic and operational scenarios at the selected stages, very useful for problems with long-term time horizons.

The rest of the paper is organized as follows: Sect. 2 introduces the main concepts and notation of the risk neutral multistage multiperiod mixed 0-1 optimization model for strategic and operational planning problems. Section 3.1 presents the Timeinconsistent Stochastic Dominance (TSD) functional for strategic functions where operational functions are embedded. Section 3.2 presents the time-consistent Expected Conditional Stochastic Dominance (ECSD) functional for operational functions. And, Sect. 4 discusses the results and outlines a future research plan.

\section{Multistage multihorizon stochastic mixed 0-1 optimization}

\subsection{Introduction}

Let us some definitions for a multistage scenario tree. A stage of a given horizon is a set of consecutive time periods where the realizations of the uncertain parameters take place. A scenario is a realization of the uncertain parameters in the stages along a time horizon. A node for a given stage in the scenario tree is the representation of a group of scenarios that have the same realization of the uncertain parameters up to the stage. The Nonanticipativity principle states that the scenarios of a group with one-to-one correspondence with a node in the tree have a unique solution up to the stage where the node belongs to.

Figure 1 depicts a scenario tree where the uncertain parameters, whose realizations as represented in a node are either strategic or operational. However, it is a bad approach 


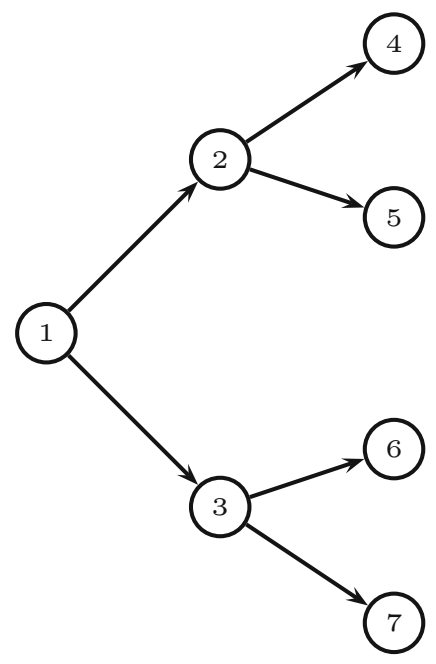

$$
\Omega=\Omega^{1}=\{4,5,6,7\} ; \Omega^{2}=\{4,5\}
$$$$
N=\{1, \ldots, 7\} ; N^{2}=\{2,3\}
$$$$
A_{3}=\{1,3\}, S_{2}=\{4,5\}
$$

Fig. 1 Multistage scenario tree

for CEP in case where a set of uncertain parameters in a node is a mixture of both types and there has been no difference in their treatment in the model.

For modeling the CEP of a system along a usually long-term, the strategic decisions in a node should not be based on the isolated realizations of operational parameters in ancestor nodes. They should basically be based on the strategic decisions made in the ancestor nodes, the strategic parameters in the node and its successors, and in the stagewise-dependent ancestor strategic parameters, if any.

The operational scenarios in a node should only be considered for the following types of decisions:

- Operational decisions for the related operational scenarios in the strategic node, on an individual basis.

- Strategic decisions to be made in the node and its ancestor strategic nodes, as a whole set.

- Operational decisions, usually, made in their immediate successor strategic nodes, for specific types of problems where the operations in the last periods of a stage may influence in the operations to be made in those successor nodes.

In this work, the structure of a multistage multihorizon scenario tree is studied, where the multistage strategic tree includes the nodes in the first periods of each stage. The multihorizon setting is related to the operational scenario graphs up to a given stage and the operational scenario two-stage trees after that stage. An operational graph structure for a strategic node is rooted with the operational replicas of the node, and it includes a set of so-named operational scenarios, each one composed of an operational node for each period in the stage. An operational two-stage tree structure for a strategic node is rooted with the related operational replica, and it includes a set of its operational scenarios for the reference period that, in a weighted form, represents all of them in the stage. The goal consists of minimizing the NPV of the expected investment cost of the CEP infrastructure elements in the set of strategic nodes plus 
the expected cost related to the operation of the available CEP elements along the time horizon. Thus, CEP is only performed in the strategic nodes, and the operation of the available elements is performed at the operational nodes. See also the two-stage structure in Kaut et al. (2014), Werner et al. (2013).

\subsection{Multistage strategic scenario tree}

Let the following notation for a multistage multiperiod strategic scenario tree, as depicted in Fig. 1.

$\mathcal{T}$, set of periods (usually, semesters, years) in the time horizon, such that $T=|\mathcal{T}|$ and $\mathcal{T}=\{1, \ldots, T\}$.

$\mathcal{E}$, set of consecutive stages in the strategic scenario tree where the set $\mathcal{T}$ is partitioned, such that $E=|\mathcal{E}|$ and $\mathcal{E}=\{1, \ldots, E\}$.

$\mathcal{T}^{e}$, set of periods in stage $e$, for $e \in \mathcal{E}$, such that $\mathcal{T}=\cup_{e \in \mathcal{E}} T^{e}$ and $\mathcal{T}^{e} \cap \mathcal{T}^{e^{\prime}}=\varnothing$ for $e, e^{\prime} \in \mathcal{E}: e \neq e^{\prime}$.

$\mathcal{N}$, set of strategic nodes, where the system's CEP is decided, such that $N=|\mathcal{N}|$ and $\mathcal{N}=\{1, \ldots, N\}$.

$\mathcal{N}^{e}$, set of strategic nodes that belong to stage $e$, for $e \in \mathcal{E}$, such that $\mathcal{N}=$ $\cup_{e \in \mathcal{E}} \mathcal{N}^{e}$ and $\mathcal{N}^{e} \cap \mathcal{N}^{e^{\prime}}=\varnothing$ for $e, e^{\prime} \in \mathcal{E}: e \neq e^{\prime}$. Without loss of generality (wlog), it is assumed that $\mathcal{N}^{1}$ is a singleton set and $1 \in \mathcal{N}^{1}$.

$\Omega$, strategic scenario set.

$\Omega_{n}$, group of strategic scenarios in set $\Omega$ with one-to-one correspondence with node $n$, for $n \in \mathcal{N}$. Note: $\Omega_{n}$ is singleton for any leaf node $n$ of the strategic scenario tree (i.e., $n \in \mathcal{N}^{E}$ ). Let us assume that $\omega=n$, for $\omega \in \Omega$.

$e(n)$, stage the scenario node $n$ belongs to, for $n \in \mathcal{N}$. Note: Wlog it is assumed that there is only one strategic node for any stage in a Hamiltonian path rooted with node 1 in set $\mathcal{N}^{1}$ until its leaf node in the scenario tree (i.e., a node in set $\mathcal{N}^{E}$ ).

$\underline{t}(e)$, first period in set $\mathcal{T}^{e}$ for stage $e$ in set $\mathcal{E}$, and $\bar{t}(e)$ is its last period.

$t(n)$, period the strategic node $n$ belongs to, for $n \in \mathcal{N}$. Wlog it is assumed that $t(n)$ is the first period in set $\mathcal{T}^{e(n)}$, so, $t(n)=\underline{t}(e(n))$.

$w^{\omega}$, weight or probability assigned to strategic scenario $\omega \in \Omega$, such that $w^{n}=$ $\sum_{\omega \in \Omega_{n}} w^{\omega}$ and $\sum_{n \in \mathcal{N} e} w^{n}=1 \forall e \in \mathcal{E}$.

$\mathcal{A}_{n}$, set composed by the same strategic node $n$ and its ancestor strategic nodes in the scenario tree, for $n \in \mathcal{N}$.

$\tilde{\mathcal{A}}_{n}$, subset of ancestor nodes to node $n$ in set $\mathcal{A}_{n}$ whose related variables have nonzero elements in constraints of node $n$, for $n \in \mathcal{N}$.

$\sigma(n)$, immediate ancestor strategic node to node $n$, for $n \in \mathcal{N}$.

$\mathcal{S}_{n}$, successor strategic set to node $n$, for $n \in \mathcal{N}$. Note: $\mathcal{S}_{n}=\emptyset$ for $n \in \mathcal{N}^{E}$.

$\mathcal{S}_{n}^{1} \subset \mathcal{S}_{n}$, immediate successor strategic set to node $n$, for $n \in \mathcal{N}$.

$\tilde{e}$, last stage where each period in set $\mathcal{T}^{e}$ is to be individually considered in the operational scenario graph structure to be presented below. Note: The graph structure becomes an operational scenario two-stage tree for any stage $e$, such that $e \in \mathcal{E}: e>\tilde{e}$, where $0 \leq \tilde{e} \leq E$. 


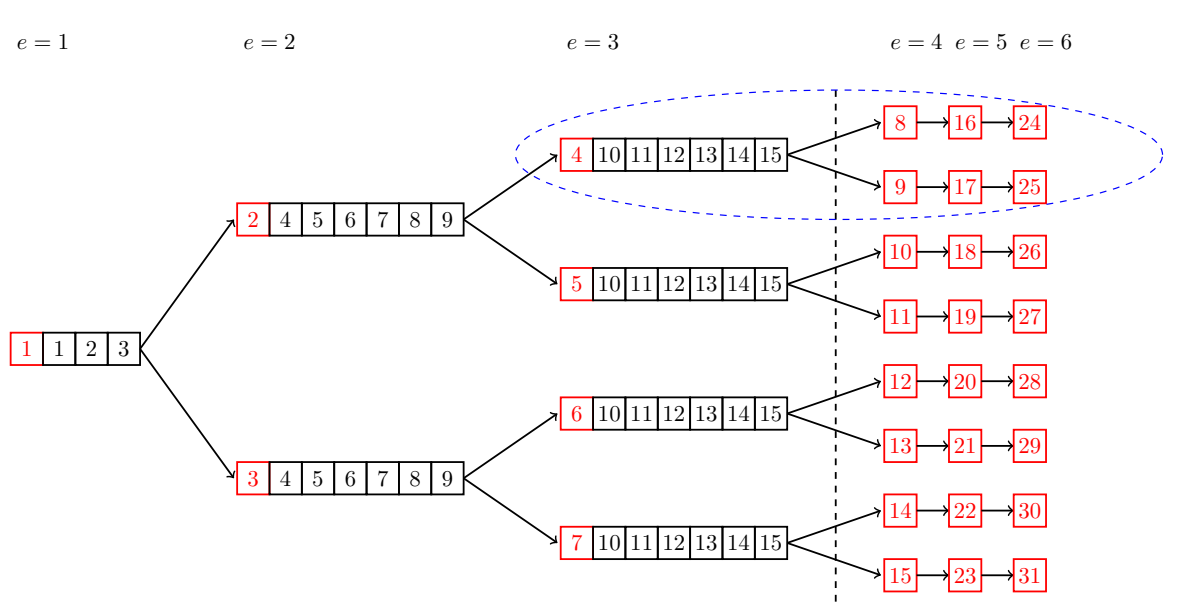

Fig. 2 A strategic scenario tree. $E=6,\left|\mathcal{T}^{e}\right|=6 \forall e \in \mathcal{E}, \tilde{e}=3, \mathrm{~N}=31$

Remark All operational nodes belong to a graph structure for $\tilde{e}=E$, and they belong to a two-stage tree structure for $\tilde{e}=0$. Notice that the multiperiod setting for the latter structure is replaced with a reference period.

Figure 2 depicts some operational nodes for the periods in set $\mathcal{T}^{e}$ for $e=1,2,3$ as well as the operational scenarios for the reference periods in the stages $\{e>\tilde{e}\}$. [Note: The first part of the figure, i.e., up to stage $e=3$ has been taken from Vespucci et al. (2013)].

\subsection{Operational structures: multiperiod graph and reference period two-stage tree}

Let us denote a operational multiperiod scenario graph in strategic node $n$ as structure graph $_{n}$, for $n \in \mathcal{N}: e(n) \leq \tilde{e}$, and an operational scenario two-stage tree as structure tree $_{n}$, for $n \in \mathcal{N}: e(n)>\tilde{e}$, see Fig. 3 .

The elements for each strategic node $n$ are as follows, for $n \in \mathcal{N}$ :

$\mathcal{Q}_{n}$, operational node set.

$\mathcal{Q}_{n}^{t}$, operational node set that belongs to period $t$ in structure $\operatorname{graph}_{n}$, for $t \in \mathcal{T}^{(n)}$, such that $\mathcal{Q}_{n}=\cup_{t \in \mathcal{T} e(n)} Q_{n}^{t}$.

By construction, $\left|\mathcal{Q}_{n}^{t(n)}\right|=\left|\mathcal{Q}_{n}^{t}\right| \forall t \in \mathcal{T}^{(n)}$ in structure graph $_{n}$, since $\mathcal{Q}_{n}^{t(n)}$ gives the realizations of the operational uncertainty in period $t(n)$ in $g r a p h_{n}$, being obviously the first period in set $\mathcal{T}^{e(n)}$.

For structure rree $_{n}, \mathcal{Q}_{n}$ is considered as the set of scenarios in the two-stage tree rooted with the unique operational replica of node $n$.

$t(q)$, period the operational node $q$ belongs to in structure $\operatorname{graph}_{n}$ (i.e., for $e(n) \in \mathcal{E}$ : $e(n) \leq \tilde{e})$ and, so, $t(q) \in \mathcal{T}^{e(n)}$, for $q \in \mathcal{Q}_{n}$. Note: $t(q)=t(n)$ for $q \in \mathcal{Q}_{n}^{t(n)}$ and, thus, $\mathcal{Q}_{n}^{t(n)}$ is the set of operationally-oriented replicas of strategic node $n$, being the root nodes in the graph structure. 
$\mathcal{L}_{n}$, leaf node set in structure $\operatorname{graph}_{n}$, that is, $\mathcal{L}_{n} \equiv \mathcal{Q}_{n}^{t}$ where $t=\bar{t}(e(n))$, for $e(n) \leq \tilde{e}$. It is a very useful set for considering variables in the operational nodes that have also nonzero elements in the constraints of the operational replicas of the nodes in the immediate successor strategic node set $\mathcal{S}_{n}^{1}$. In our case, the replicas of any of those successor nodes are, precisely, the root ones of the related immediate successor operational structures $\operatorname{graph}_{n^{\prime}}$ for $e(n)<\tilde{e}$ and operational structures two-stage $\operatorname{tree}_{n^{\prime}}$ for $e(n)=\tilde{e}$, for $n^{\prime} \in \mathcal{S}_{n}^{1}$. Remember that in the tree structure there it only one replica of the node.

As an illustrative example, let us consider an energy generation CEP where the water in hyper hydro reservoirs as well as gas, oil and other crucial raw materials in thermal plants that are stored at the end of a period are used in the next one. So, the items that are stored, say, at the end of any leaf node in a stage become available for use at the first period of the next one. On contrary, those items are assumed to be non-stored for strategic nodes in stage $e \in \mathcal{E}$, for $e(n)>\tilde{e}$. On the other hand, if the storing of those items in the system does not play a significant role in the system's operation then it is assumed that $\tilde{e}=0$ and, thus, only structure tree $_{n}$ is taken into account, for any $n \in \mathcal{N}$.

$\Pi_{n}$, operational scenario set $\{\pi\}$. For notational purposes, consider in structure $\operatorname{graph}_{n}$ that $\pi \equiv q$ for $q \in \mathcal{Q}_{n}^{t}$ where $t=\bar{t}(e(n))$ (i.e., the last period in stage $e(n))$. Consider also in structure $\operatorname{tree}_{n}$ that $\mathcal{Q}_{n}=\Pi_{n}$, following the rationale considered above.

$\mathcal{A}_{n}^{q}$, ancestor operational node set (including itself) of operational node $q$ in structure $\operatorname{graph}_{n}$, for $q \in \mathcal{Q}_{n}$, such that $\mathcal{A}_{n}^{\pi}$ is the set of ancestor nodes (including itself) of operational scenario $\pi$ for $\pi \in \Pi_{n}$. Notice that strategic node $n$ is not in $\mathcal{A}_{n}^{q}$ for $q \in \mathcal{Q}_{n}$, but remember that $t(q)=t(n)$ for its replicas $q \in \mathcal{Q}_{n}^{t(n)}$, see Fig. 3. Note: $\mathcal{A}_{n}^{q}=\{q\}, q \in \mathcal{Q}_{n}^{t(n)}$.

For easing modeling notation, it is considered that $\mathcal{A}_{n}^{\pi}=\{\pi\}$, for $\pi \in \Pi_{n}$ in structure $\operatorname{tree}_{n}$.

$\sigma(q)$, immediate ancestor operational node to operational node $q$ in structure $g r a p h_{n}$, for $q \in \mathcal{Q}_{n}$. Note: $\sigma(q)=\emptyset$, for $q \in \mathcal{Q}_{n}^{t(n)}$.

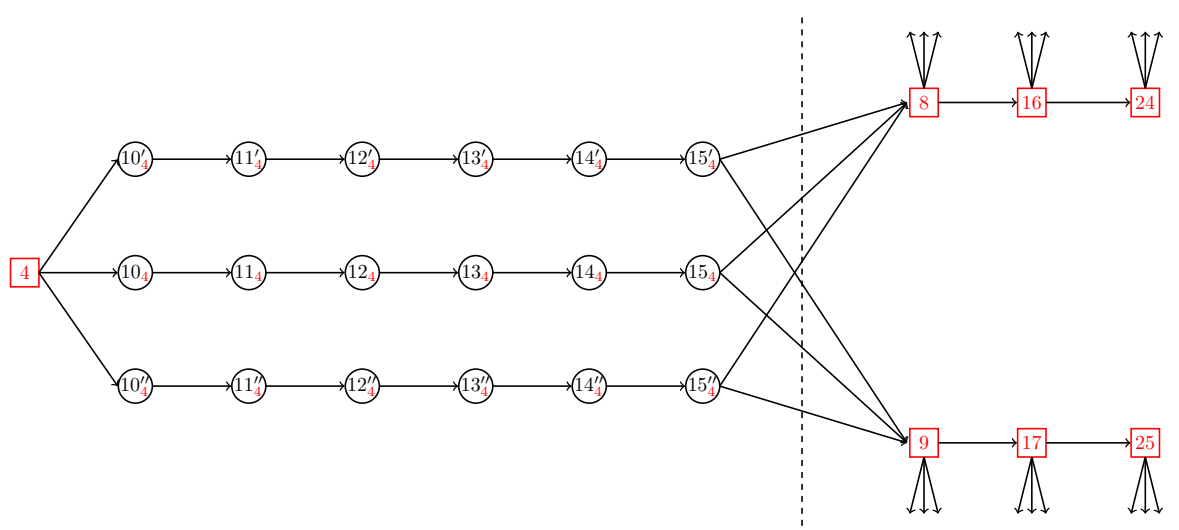

Fig. 3 Operational multiperiod graph $_{4}$ and operational reference period tree $_{n}$ for $n \in \mathcal{S}_{4}$ 
$w^{\pi}$, weight or probability of operational scenario $\pi$, for $\pi \in \Pi_{n}$, in both structures graph $_{n}$ and tree $_{n}$, such that $\sum_{\pi \in \Pi_{n}} w^{\pi}=1$. Note: Given the character of structure $\operatorname{graph}_{n}$, it results that $w^{q}=w^{\pi} \forall q \in \mathcal{A}_{n}^{\pi}$.

For clarification purposes, let Fig. 3 be a zoom of Fig. 2 for strategic node $n=4$ and successors. Then, the operational uncertainty is represented in $\operatorname{graph}_{n}$, where stage $e(4)=3$ and the node set $\mathcal{L}_{4}=\left\{15_{4}^{\prime}, 15_{4}, 15_{4}^{\prime \prime}\right\}$ reaches the operational scenario sets $\Pi_{8}$ and $\Pi_{9}$. By construction, since $e(8)=e(9)=4>\tilde{e}=3$, observe that the root of the operational scenario two-stage $t_{r e e_{n}}$ is, precisely, the own replica of strategic node $n$, for $n=\{8,9\}$. Notice that their related second stages are represented by the node sets $\Pi_{8}$ and $\Pi_{9}$. Observe also that there is no difference in the treatment of the uncertainty in the periods that belong to set $T^{e(n)}$, since at that point in time no variables are considered linking the periods. Then, it is assumed that the operational scenarios occur in the reference period and, so, it is assumed that set $T^{e(n)}$ is represented by the related reference period.

On the other hand, observe in Fig. 2 that strategic node $n=4$ belongs to the immediate successor strategic node set $\mathcal{S}_{2}^{1}$. Since $e(4)$ is not greater than $\tilde{e}=3$ (in fact, it is equal), the related operational uncertainty is represented in $g r a p h_{n}$ as depicted in Fig. 3, where $\mathcal{Q}_{4}=\cup_{t \in \mathcal{T}^{3}} \mathcal{Q}_{4}^{t}$. Notice also that it is assumed that there are linking variables between two consecutive periods in $\mathcal{T}^{3}=\{10, \cdots, 15\}$. Remember that any strategic node $n$ for $e(n) \leq \tilde{e}$ is replaced with as many operational replicas as the number $\left|\Pi_{n}\right|$ of the operational scenarios. For example, strategic node $n=4$ (whose period is $t(n)=10$ ) is replaced with the operational replicas $10_{4}^{\prime}, 10_{4}$ and $10_{4}^{\prime \prime}$, so, $\Pi_{4}^{\pi}=\left\{15_{4}^{\prime}, 15_{4}, 15_{4}^{\prime \prime}\right\}$, such that as an illustration $\mathcal{A}_{4}^{\pi}=\left\{10_{4}, 11_{4}, 12_{4}, 13_{4}, 14_{4}, 15_{4}\right\}$, for $\pi=15_{4}$.

For purposes of completion, Fig. 4 depicts a two-stage tree structure for the strategic multistage tree depicted in Fig. 1.

\subsection{Infrastructure elements in a synthesized CEP problem}

The notation of the elements in the system's CEP is as follows:

$\mathcal{I}$, set of infrastructure elements in the system whose capacity could be independently constructed or expanded.

$\mathcal{I}^{I}$, subset of infrastructure elements in set $\mathcal{I}$, where $\hat{x}_{i}$ denotes the capacity of element $i$, and the value 1 of the step $0-1$ variable $\left(x^{n}\right)_{i}$ means that it is constructed by period $t(n)$ in strategic node $n$ and otherwise, 0 , for $i \in \mathcal{I}^{I}, n \in \mathcal{N}$.

Illustrative examples are some types of power generation units as thermal plants and hydropower turbines in energy generation, cables in electricity transmission lines, and stations and hedges in rapid transit networks. Other examples are production units, machines, transportation capacity, distribution, sorting and market centers in supply chains, and others.

$\mathcal{I}^{C}$, subset of infrastructure elements in set $\mathcal{I}$, where the step continuous variable $\left(y^{n}\right)_{i}$ denotes the capacity of element $i$ that is built by period $t(n)$ in strategic node $n$, up to a given bound, say $\hat{y}_{i}$, for $i \in \mathcal{I}^{C}, n \in \mathcal{N}$. Notice that the 


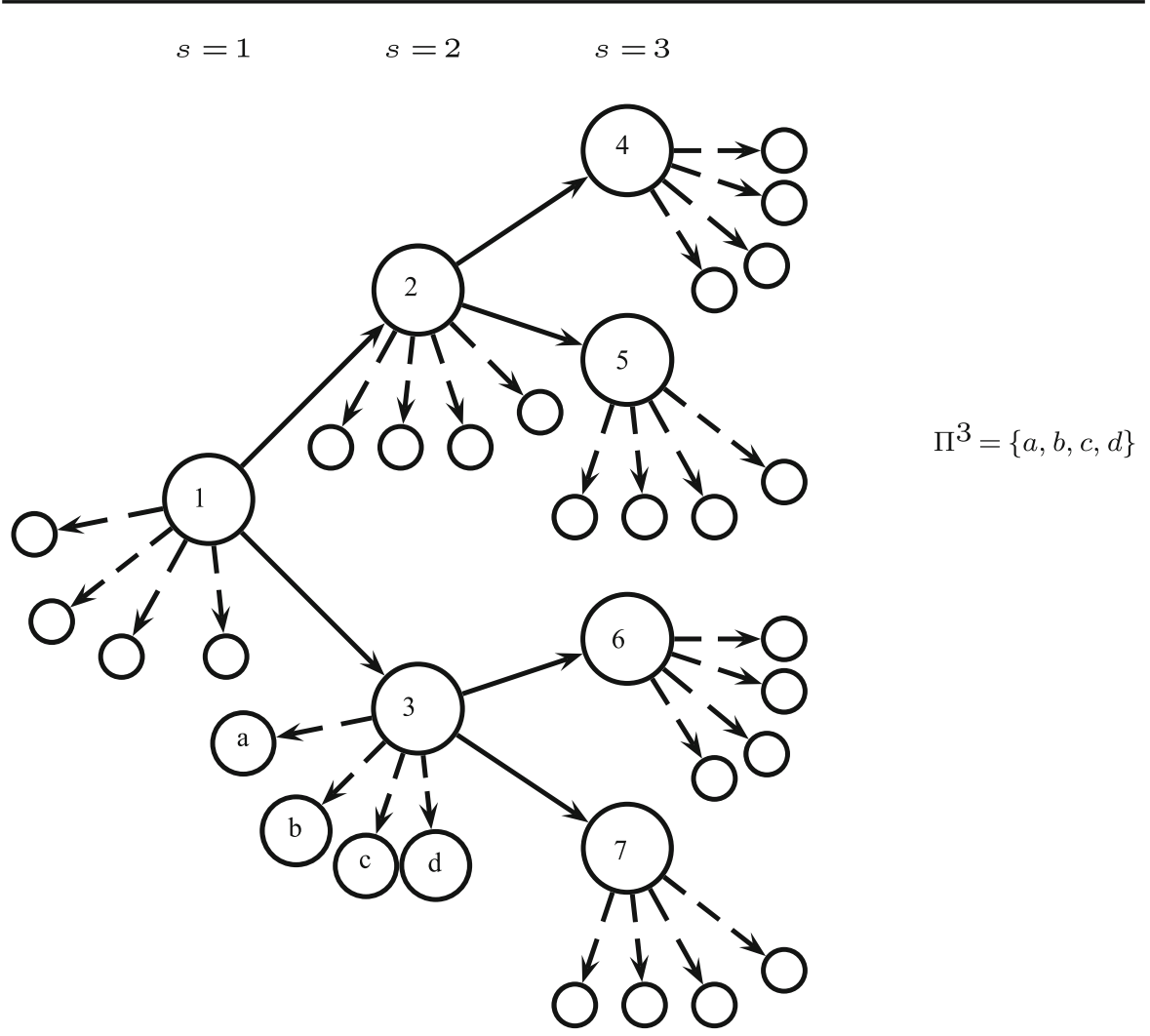

Fig. 4 Reference period two-stage trees for strategic nodes. $\tilde{e}=0$

capacity can be increased in any successor strategic node to the node where it was constructed.

Illustrative examples are intermittent power generation types as wind, solar and photovoltaic farms, among others. Note: $\mathcal{I}=\mathcal{I}^{I} \cup \mathcal{I}^{C}$

$\mathcal{I}_{i}$, subset of elements in set $\mathcal{I}$, whose construction or capacity increase cannot start until the construction or the last capacity increase of element $i$ is over, for $i \in \mathcal{I}^{I}$ in the first case and $i \in \mathcal{I}^{C}$ in the second one.

$\mathcal{J}$, set of operational elements in the system.

Illustrative examples are energy generation in the plants, energy flow though a cable of a transmission line, and passengers transportation flow from one station to another in a rapid transit network. Other examples are product production in a factory, plant or unit, water stored in reservoirs in a hydro-power system, raw material, subassemblies and end-products stored in warehouses, and others.

$\mathcal{I}^{j}$, set of infrastructure elements in set $\mathcal{I}$ that should be available when operational element $j$ is active, for $j \in \mathcal{J}$.

$\tau_{i}$, latency (number of periods) required between the one where the construction of element $i$ starts for $i \in \mathcal{I}^{I}$ or the period where the last capacity increase 
is performed for $i \in \mathcal{I}^{C}$, and the period at which the element becomes available.

$\kappa_{i}$, earliest period to start the construction of infrastructure element $i$ that satisfies the precedence relationships given by set $\left\{i^{\prime} \in \mathcal{I}: i \in \mathcal{I}_{i^{\prime}}\right\}$, for $i \in \mathcal{I}$. It is computed as

$$
\kappa_{i}=\max _{i^{\prime} \in \mathcal{I}: i \in \mathcal{I}_{i^{\prime}}}\left\{\kappa_{i^{\prime}}+\tau_{i^{\prime}}\right\} .
$$

$\kappa^{j}$, earliest period at which operational element $j$ can be active and, then, it satisfies the precedence relationships given by set $\mathcal{I}^{j}$, for $j \in \mathcal{J}$. It is computed as

$$
\kappa^{j}=\max _{i \in \mathcal{I}^{j}}\left\{\kappa_{i}+\tau_{i}\right\} .
$$

$\iota(t(q))_{i}$, strategic node whose period $t\left(\iota(t(q))_{i}\right)$ is the latest one by which infrastructure element $i$, for $i \in \mathcal{I}$, can start its construction, so that it is available for use in the system at period $t(q)$ in structure $\operatorname{graph}_{n}$, so, $q \in \mathcal{Q}_{n}, n \in \mathcal{N}: \kappa_{i} \leq t(n) \wedge e(n) \leq \tilde{e}$.

Node $\iota(t(q))_{i}$ is computed as

$$
\iota(t(q))_{i}=\operatorname{argmax}_{n^{\prime} \in \mathcal{A}_{n}}\left\{t\left(n^{\prime}\right) \in \mathcal{T}: t\left(n^{\prime}\right) \leq t(q)-\tau_{i}\right\}
$$

On the other hand, the concept of $\iota(t(q))_{i}$ for operational scenario $q$ in structure $\operatorname{tree}_{n}$ is as for the graph structure, such that $q \in \mathcal{Q}_{n}, n \in \mathcal{N}: \kappa_{i} \leq t(n) \wedge e(n)>\tilde{e}$, where $\mathcal{Q}_{n} \equiv \Pi_{n}$. However for computing $\iota(t(q))_{i}$, period $t(q)$ is replaced with $t(n)$ in expression (1), i.e., the computing of the latency for structure tree $e_{n}$ only considers the first periods of the stages.

Remark 1 All operational nodes $\{q\}$ in the sets $\mathcal{Q}_{n}$ share the same node $\iota(t(q))_{i}$ for the strategic nodes $\{n\}$ that belong to the same stage, say $e$, such that $n \in \mathcal{N}^{e}$, for $e \in \mathcal{E}$.

Remark 2 The operational nodes $\{q\}$ in set $\mathcal{Q}_{n}$, for $n \in \mathcal{N}$, that share the same node $\iota(t(q))_{i}$ are those that have in common the same stage $e$ for which period $t(q)-\tau_{i}$ belongs to set $\mathcal{T}^{e}$, for $e \in \mathcal{E}$.

\subsection{Risk neutral (RN) model}

Formally, the value 1 of step $0-1$ variable $\left(x^{n}\right)_{i}$, for $i \in \mathcal{I}^{I}$, means that infrastructure element $i$ has started its construction by period $t(n)$ (i.e., it started at strategic node $n$ or at any of its ancestor in the scenario tree), and otherwise, 0 , for $n \in \mathcal{N}: \kappa_{i} \leq$ $t(n) \leq T-\tau_{i}$, whose capacity is $\hat{x}_{i}$.

Formally, a positive value of the step continuous variable $\left(y^{n}\right)_{i}$, for $i \in \mathcal{I}^{C}$, means that infrastructure element $i$ has started its construction with that capacity or it started the last increase to reach that capacity by period $t(n)$ (i.e., at strategic node $n$ or at any of its ancestor in the scenario tree), and otherwise, 0 , for $n \in \mathcal{N}: \kappa_{i} \leq t(n) \leq T-\tau_{i}$. Remember that the capacity $\left(y^{n}\right)_{i}$ is allowed to grow up at any strategic node $n^{\prime}$, for $n^{\prime} \in \mathcal{N}: t(n)<t\left(n^{\prime}\right) \leq T-\tau_{i}$ up to the given bound $\hat{y}_{i}$. 
Let the impulse $0-1$ variable $\left(\delta^{n}\right)_{i}$, such that its value 1 means that infrastructure element $i$, for $\mathcal{I}^{C}$, starts its construction or increases its capacity at period $t(n)$ in strategic node $n$ and otherwise, 0 , for $n \in \mathcal{N}: \kappa_{i} \leq t(n) \leq T-\tau_{i}$. Let $\underline{y}_{i}$ and $\bar{y}_{i}$ denote the conditional lower and upper bound of each capacity increase of element $i$, res., such that 'conditional' means that the increase happens if and only if $\left(\delta^{n}\right)_{i}=1$, see below.

Let the impulse continuous variable $\left(z^{q}\right)_{j}$ denote, for $j \in \mathcal{J}$, the activity at operational node $q$ in structure $\operatorname{graph}_{n}$ for $e(n) \leq \tilde{e}$ and the activity at for operational scenario $q$ in structure $\operatorname{tree}_{n}$ for $e(n)>\tilde{e}$, for $q \in \mathcal{Q}_{n}, n \in \mathcal{N}$. Remark: Any impulse variable $\left(z^{q}\right)_{j}$ cannot have a value greater than zero if all related step variables $\left(x^{l(t(q))_{i}}\right)_{i}$ for $i \in \mathcal{I}^{I} \cap \mathcal{I}^{j}$ and $\left(y^{l(t(q))_{i}}\right)_{i}$ for $i \in \mathcal{I}^{C} \cap \mathcal{I}^{j}$ do not have nonzero values.

So, let $x^{n}$ and $\hat{x}$ denote the vectors of the variables $\left(x^{n}\right)_{i}$ and capacity bounds $\hat{x}_{i}$, res., for $i \in \mathcal{I}^{I} ; y^{n}$ and $\hat{y}$ are the vectors of the variables $\left(y^{n}\right)_{i}$ and capacity bounds $\hat{y}_{i}$, res., for $i \in \mathcal{I}^{C}$; and $z^{q}$ is the vector of the variables $\left(z^{q}\right)_{j}$ for $j \in \mathcal{J}$.

The other parameters of the synthesized RN model for strategic node $n \in \mathcal{N}$ are as follows:

$a_{1}^{n}$ (res. $c_{1}^{n}$ ), vector of objective function coefficients for the strategic variables in vector $x^{n}$ (res. $\left.y^{n}\right)$.

$b_{1}^{q}$, vector of objective function coefficients for the operational variables in vector $z^{q}$, for $q \in \mathcal{A}_{n}^{\pi}, \pi \in \Pi_{n}, n \in \mathcal{N}$. Note: Remember that it is assumed that $\mathcal{A}_{n}^{\pi}=\{\pi\}$ in structure tree $_{n}$, for $\pi \in \Pi_{n}$.

$h^{n}$ (res. $h^{q}$ ), rhs for the constraints related to strategic node $n$ (res. operational node $q$, for $\left.q \in \mathcal{Q}_{n}\right)$.

$A_{n}^{n^{\prime}}$ (res. $C_{n}^{n^{\prime}}$ ), constraint matrix for the strategic variables in vector $x^{n^{\prime}}$ (res. $y^{n^{\prime}}$ ) of the ancestor strategic node $n^{\prime}$ in strategic constraints related to node $n$, for $n^{\prime} \in \tilde{\mathcal{A}}_{n}$.

$B_{n}^{\ell}$, constraint matrix for the operational variables in leaf vector $y^{\ell}$ in the constraints related to operational node $q$ in structure $\operatorname{graph}_{n}$, for $\ell \in \mathcal{L}_{\sigma(n)}: e(n) \leq$ $\tilde{e}, q \in \mathcal{Q}_{n}: t(q)=t(n)$. On the other hand, $B_{n}^{\ell}$ is the constraint matrix for the operational scenario $q$ in structure $\operatorname{tree}_{n}$, for $\ell \in \mathcal{L}_{\sigma(n)}: e(n)=\tilde{e}+1, q \in \mathcal{Q}_{n}$.

$B_{q}^{\sigma(q)}$, constraint matrix for the operational variables in vector $z^{\sigma(q)}$ in the constraints related to operational node $q$ in structure $\operatorname{graph}_{n}$, for $q \in \mathcal{Q}_{n}: e(n) \leq$ $\tilde{e} \wedge t(q)>t(n)$.

$B^{q}$, constraint matrix for the operational variables in vector $z^{q}$ in the constraints related to operational node $q$, for $q \in \mathcal{Q}_{n}: e(n) \leq \tilde{e}$. On the other hand, $B^{q}$ is the constraint matrix for the operational scenario $q$ in structure $\operatorname{tree}_{n}$, for $q \in \Pi_{n}: e(n)>\tilde{e}$.

Remember that $\mathcal{Q}_{n}=\cup_{t \in \mathcal{T}^{e(n)}} Q_{n}^{t}$ in $\operatorname{graph}_{n}$ (i.e., $e(n) \leq \tilde{e}$ ) and, for simplifying modeling purposes, $\mathcal{Q}_{n}=\Pi_{n}$ and $\mathcal{A}_{n}^{\pi}=\{\pi\}$ in $\operatorname{tree}_{n}$ (i.e., $\left.e(n)>\tilde{e}\right)$. 
The objective function can be expressed as

$$
\begin{aligned}
& \min \sum_{i \in \mathcal{I}} \sum_{n \in \mathcal{N}: \kappa_{i} \leq t(n) \leq T-\tau_{i}} \frac{1}{\left(1+\rho^{t(n)}\right)} w^{n}\left[\left(a_{1}^{n}\left(x^{n}-x^{\sigma(n)}\right)\right)_{: i \in \mathcal{I}^{I}}\right. \\
& \left.+\left(c_{1}^{n}\left(y^{n}-y^{\sigma(n)}\right)\right)_{: i \in \mathcal{I}^{C}}\right] \\
& +\sum_{n \in \mathcal{N}} \frac{1}{\left(1+\rho^{t(n)}\right)} w^{n}\left(\left|\mathcal{T}^{e(n)}\right|_{: e(n)>\tilde{e})} \sum_{\pi \in \Pi_{n}} \sum_{q \in \mathcal{A}_{n}^{\pi}} w^{\pi} b_{1}^{q} z^{q},\right.
\end{aligned}
$$

where $\rho^{t(n)}$ is the interest rate in period $t(n)$.

The objective function (2) consists of minimizing the NPV of the following two terms. The first one is the expected cost of the CEP investment in the infrastructure elements. Notice that $x^{n}-x^{\sigma(n)}=1$ for $\in \mathcal{I}^{I}$ means that element $i$ has started its construction at period $t(n)$. Similarly, $y^{n}-y^{\sigma(n)}>0$ for $i \in \mathcal{I}^{C}$ means that element $i$ either has started its construction at period $t(n)$ or its capacity has been increased at that node.

The second term of function (2) is the expected cost of the operational elements (or activities). Notice that the cost in the operational scenarios in $\Pi_{n}$ is related to the reference period in the two-stage tree. It is weighted with the number of periods $\left|\mathcal{T}^{e(n)}\right|$ in the related stage $e(n)$. On the contrary, structure $g r a p h_{n}$ applies for $e(n) \leq \tilde{e}$ and, then, the cost in each scenario $\pi$ in set $\Pi_{n}$ is the sum of the costs in the related ancestor operational nodes in set $\mathcal{A}_{n}^{\pi}$, each one being related to a period in set $\mathcal{T}^{e(n)}$.

The set of strategic constraints can be expressed as

$$
\begin{array}{cl}
\left(x^{\sigma(n)}\right)_{i} \leq\left(x^{n}\right)_{i}, \quad\left(x^{n}\right)_{i} \in\{0,1\} & \forall n \in \mathcal{N}: \kappa_{i} \leq t(n) \leq T-\tau_{i}, i \in \mathcal{I}^{I} \\
\left(x^{n}\right)_{i^{\prime}}-\left(x^{\sigma(n)}\right)_{i^{\prime}} \leq\left(x^{l(t(n))_{i}}\right)_{i} & \forall n \in \mathcal{N}: \kappa_{i^{\prime}} \leq t(n) \leq T-\tau_{i^{\prime}}, i^{\prime} \in \mathcal{I}_{i}, i \in \mathcal{I}^{I} \\
0 \leq\left(y^{n}\right)_{i} \leq \hat{y}_{i},\left(\delta^{n}\right)_{i} \in\{0,1\}, & \\
\underline{y}_{i} \cdot\left(\delta^{n}\right)_{i} \leq\left(y^{n}\right)_{i}-\left(y^{\sigma(n)}\right)_{i} \leq \bar{y}_{i} \cdot\left(\delta^{n}\right)_{i} & \forall n \in \mathcal{N}: \kappa_{i} \leq t(n) \leq T-\tau_{i}, i \in \mathcal{I}^{C} \\
\left(y^{n}\right)_{i^{\prime}}-\left(y^{\sigma(n)}\right)_{i^{\prime}} \leq M_{i, i^{\prime}} \cdot\left(y^{l(t(n))_{i}}\right)_{i} & \forall n \in \mathcal{N}: \kappa_{i^{\prime}} \leq t(n) \leq T-\tau_{i^{\prime}}, i^{\prime} \in \mathcal{I}_{i}, i \in \mathcal{I}^{C} \\
\sum_{n^{\prime} \in \tilde{\mathcal{A}}_{n}}\left(A_{n}^{n^{\prime}} x^{n^{\prime}}+C_{n}^{n^{\prime}} y^{n^{\prime}}\right)=h^{n} & \forall n \in \mathcal{N} .
\end{array}
$$

Constraints (3) define the step variables in vector $x^{n}$. Constraints (4) allow the starting of the construction of the one-shot infrastructure elements, provided the availability of all of its precedent elements and otherwise, 0 .

Constraints (5) define the step variables in vector $y^{n}$ and force the conditional lower and upper bounds for each capacity increase (even, for the first one). The type of infrastructure elements that are considered are those whose construction and capacity increase can be performed in a continuous form for each block. Notice that $\left(\delta^{n}\right)_{i}=0$ implies $\left(y^{n}\right)_{i}=\left(y^{\sigma(n)}\right)_{i}$ (i.e., no capacity increase on element $i$ at node $n$ ).

It is assumed in constraints (6) that any capacity increase in element $i^{\prime}$ in set $\mathcal{I}_{i}$ cannot be greater than the $M_{i, i^{\prime}}$-factorized capacity of its precedent element $i$, 
provided that it is available and otherwise, 0 . Note: The problem-dependent fraction $M_{i, i^{\prime}}$ gives the impact of the capacity of element $i$ in the capacity increase of element $i^{\prime}$, for $i^{\prime} \in \mathcal{I}_{i}, i \in \mathcal{I}^{C}$. Constraints (7) define the general relationships among the infrastructure elements at each strategic node.

The set of constraints linking strategic and operational variables can be expressed as

$$
\begin{array}{cl}
0 \leq\left(z^{q}\right)_{j} \leq N_{i, j} \cdot \hat{x}_{i}\left(x^{\iota(t(q))_{i}}\right)_{i} & \forall q \in \mathcal{Q}_{n}, n \in \mathcal{N}: \kappa_{i} \leq t(n), j \in \mathcal{J}_{i}, i \in \mathcal{I}^{I} \\
0 \leq\left(z^{q}\right)_{j} \leq N_{i, j} \cdot\left(y^{l(t(q))_{i}}\right)_{i} & \forall q \in \mathcal{Q}_{n}, n \in \mathcal{N}: \kappa_{i} \leq t(n), j \in \mathcal{J}_{i}, i \in \mathcal{I}^{C} \\
\left(z^{q}\right)_{j}=0 & \forall q \in \mathcal{Q}_{n}, n \in \mathcal{N}: t(n)<\kappa^{j}, j \in \mathcal{J}_{i}, i \in \mathcal{I} .
\end{array}
$$

Constraints (8) and (9) allow operational elements be active, provided the availability of all of their related influential infrastructure elements and otherwise, 0 . The problem-dependent fraction $N_{i, j}$ gives the impact of the capacity of element $i$ in the operational variable $\left(z^{q}\right)_{j}$, for $j \in \mathcal{J}_{i}, i \in \mathcal{I}$. Illustrative examples are passenger demand at a given station that cannot be attended until the station and its connections are available in rapid transit networks, and energy generation in a power plant that cannot be possible until the plant is available. Other examples are energy flow through a cable of a transmission system that cannot be possible until the cable is available, product production in a factory that cannot be possible until the related infrastructure elements are available in a given supply chain network, etc.

The set of constraints for allowing the operations in the available infrastructure elements in the system for strategic node $n$, for $n \in \mathcal{N}^{e}, e \in \mathcal{E}$, can be expressed as

$$
\begin{aligned}
& \left(\sum_{\ell \in \mathcal{L}_{\sigma(n): t(q)=t(n)}} w^{\ell} B_{n}^{\ell} z^{\ell}\right)+\left(B_{q}^{\sigma(q)} z^{\sigma(q)}\right): t(q)>t(n)+B^{q} z^{q}=h^{q} \quad \forall q \in \mathcal{Q}_{n}: e(n) \leq \tilde{e} \\
& \left(\sum_{\ell \in \mathcal{L}_{\sigma(n): e(n)=\tilde{e}+1}} w^{\ell} B_{n}^{\ell} z^{\ell}\right)+B^{\pi} z^{\pi}=h^{\pi} \quad \forall \pi \in \Pi_{n}: e(n)>\tilde{e} .
\end{aligned}
$$

Constraints (11) are related to structure $\operatorname{graph}_{n}$ where, by construction, there are links between the activities in the periods. Some variables in the leaf nodes related to the immediate ancestor strategic node $\sigma(n)$ have nonzero elements in the constraints of the operational nodes that are the replicas of node $n$. In order to make the model more manageable, it is worth to pointing out that the influence of the activity $z^{\ell} \forall \ell \in \mathcal{L}$ of the leaf nodes in the replicas of the successor strategic nodes is approximated by its expected value $\sum_{\ell \in \mathcal{L}_{\sigma(n)}: t(q)=t(n)} w^{\ell} B_{n}^{\ell} z^{\ell}$. For the other periods in stage $e(n)$ (i.e., subset $\left.\mathcal{T}^{e(n)}: t(q)>t(n)\right)$ the link only exists between consecutive periods (i.e., a link between operational node $q$ and its immediate ancestor $\sigma(q))$ in the stage. Typical examples of variables in vector $z^{q}$ are product storing in supply chains and production planning, water, gas and fuel storing in energy generation plants, etc. It can be assumed that the variables' linking after stage $\tilde{e}+1$ may not be relevant enough to take it into account in the decision making at early periods along the time horizon. 
Constraints (12) are related to structure tree $_{n}$ where, by construction, it is assumed that there is not any linking between the activities in the periods of a stage. Thus, the uncertainty in the operational parameters is realized in the set of operational scenarios for the reference period. Typical examples of the type of settings for $\tilde{e}=0$ are passengers' flow through a set of stations in rapid transit networks, energy generation in intermittent renewable energy sources, energy flow in transmission lines and other procurement services. Finally, observe the expected value of the terms related to the linking variables between the operational leaves of the immediate ancestor strategic node and the operational scenarios in set $\Pi_{n}$ for stage $e(n)=\tilde{e}+1$.

\section{Risk averse dynamic stochastic dominance (SD) functionals}

The popular risk neutral (RN) model (2)-(12) minimizes the expected cost in the scenarios along the time horizon. However, it ignores the variability of the objective function value in the scenarios, in particular, the impact of the solution in the "right" tail of the non-wanted scenarios (i.e., low-probability high cost scenarios). On the other hand, there is not any other function to consider, besides the objective one. There are some risk averse approaches that additionally deal with risk management (Pflug and Römisch 2007), see a computational comparison in Alonso-Ayuso et al. (2014). Among them, the measures considered in the section reduce the risk of the negative impact of the solutions in non-wanted scenarios in a better way than the others under some circumstances. See in Dentcheva and Ruszczynski (2003) its theoretical foundations, among others.

\subsection{Time-inconsistent SD measure for strategic functions}

The time stochastic dominance (TSD) risk averse measure that we have proposed elsewhere (Escudero et al. 2016, 2017a,b) is considered in our approach. As RN it also aims to minimizing the objective function expected value. However, additionally, a modeler-driven set of thresholds on the value of given functions up to each strategic node in selected stages is taken into account. The new parameters are a bound on the surplus on reaching each threshold, a bound on the probability of having surplus and a bound on the expected surplus in the stages. Hence, new variables and constraints have to be considered in the model, such that the nice scenario node-based structure of the constraint system is destroyed. The proposed risk averse measure is a mixture of the first- and second-order stochastic dominance functionals induced by integer-linear recourse for the two-stage setting introduced in Gollmer et al. (2008, 2011), res.

TSD requires the following sets of modeler-driven functions and profiles:

$\mathcal{F}^{S t}$, set of strategic functions to consider, such that the function indexed with $f$ is to be named from now on as simply function $f$. Function $f=1$ is the one to be minimized, e.g., investment and operational costs. Other functions could be related to environmental and cultural targets, etc.

$\mathcal{E}_{f}$, subset of stages in set $\mathcal{E}$, where TSD is to be considered for function $f$, for $f \in \mathcal{F}^{S t}$. 
$a_{f}^{n}$ (res. $c_{f}^{n}$ ), vector of coefficients in function $f$ for the strategic variables in vector $x^{n}$ (res. $y^{n}$ ), for $n \in \mathcal{N}, f \in \mathcal{F}^{S t}$.

$b_{f}^{q}$, vector of coefficients in function $f$ for the operational variables in vector $z^{q}$, for $q \in \mathcal{A}_{n}^{\pi}, \pi \in \Pi_{n}, n \in \mathcal{N}, f \in \mathcal{F}^{S t}$. Note: Very frequently there are zero coefficients in the vectors $a_{f}^{n}, c_{f}^{n}$ and $b_{f}^{q}$ for some functions $f$.

$\mathcal{P}_{f}^{e}$, set of profiles, for $e \in \mathcal{E}_{f}, f \in \mathcal{F}^{S t}$.

For each profile $p \in \mathcal{P}_{f}^{e}$ in stage $e \in \mathcal{E}_{f}$ for function $f \in \mathcal{F}^{S t}$, let the following modeler-driven parameters:

$\phi^{p}$, function $f$ threshold to consider up to strategic node $n$, where the operational scenarios in set $\Pi_{n}$ are taken into account, for $n \in \mathcal{N}^{e}$.

$\tilde{s}^{p}$, upper bound of the surplus of the $\pi$-expected value of function $f$ on reaching threshold $\phi^{p}$ up to strategic node $n$, for $n \in \mathcal{N}^{e}$, where the set of operational scenarios $\Pi_{n}$ is taken into account.

$\bar{s}^{p}$, upper bound of the $(n, \pi)$-expected surplus on reaching threshold $\phi^{p}$ up to stage $e$.

$\bar{v}^{p}$, upper bound of the expected fraction of the strategic nodes in set $\mathcal{N}^{e}$ with surplus on reaching threshold $\phi^{p}$.

The variables for pair $(n, p)$, where $n$ is a strategic node and $p$ is the indexation of a profile in TSD stage $e$, for $n \in \mathcal{N}^{e}, p \in \mathcal{P}_{f}^{e}, e \in \mathcal{E}_{f}$ are as follows for function $f$, for $f \in \mathcal{F}^{S t}$ :

$s^{n, p}$, continuous variable that takes the surplus of the expected value of function $f$ over threshold $\phi^{p}$ up to strategic node $n$, where the set of operational scenarios $\Pi_{n}$ is taken into account.

$v^{n, p}, 0-1$ variable such that its value is 1 if $s^{n, p}>0$ and otherwise, 0 ; and viceversa.

The TSD constraint system can be expressed as

$$
\begin{aligned}
& \sum_{i \in \mathcal{I}} \sum_{n^{\prime} \in \mathcal{A}_{n}: K_{i} \leq t\left(n^{\prime}\right) \leq T-\tau_{i}} \frac{1}{\left(1+\rho^{t\left(n^{\prime}\right)}\right)}\left[\left(a_{f}^{n^{\prime}}\left(x^{n^{\prime}}-x^{\sigma\left(n^{\prime}\right)}\right)\right)_{: i \in \mathcal{I}^{I}}+\left(c_{f}^{n^{\prime}}\left(y^{n^{\prime}}-y^{\sigma\left(n^{\prime}\right)}\right)\right)_{: i \in \mathcal{I}^{C}}\right] \\
& +\sum_{n^{\prime} \in \mathcal{A}_{n}} \frac{1}{\left(1+\rho^{t\left(n^{\prime}\right)}\right)}\left(\left|\mathcal{T}^{e\left(n^{\prime}\right)}\right|_{\left.: e\left(n^{\prime}\right)>\tilde{e}\right)} \sum_{\pi \in \Pi_{n^{\prime}}} \sum_{q \in \mathcal{A}_{n^{\prime}}^{\pi}} w^{\pi} b_{f}^{q} z^{q}-s^{n, p} \leq \phi^{p}\right. \\
& \forall n \in \mathcal{N}^{e}, p \in \mathcal{P}_{f}^{e}, e \in \mathcal{E}_{f}, f \in \mathcal{F}^{S t} \\
& 0 \leq s^{n, p} \leq \tilde{s}^{p} v^{n, p}, \quad v^{n, p} \in\{0,1\} \quad \forall n \in \mathcal{N}^{e}, p \in \mathcal{P}_{f}^{e}, e \in \mathcal{E}_{f}, f \in \mathcal{F}^{S t} \\
& \sum_{n \in \mathcal{N}^{e}} w^{n} s^{n, p} \leq \bar{s}^{p} \quad \forall p \in \mathcal{P}_{f}^{e}, e \in \mathcal{E}_{f}, f \in \mathcal{F}^{S t} \\
& \sum_{n \in \mathcal{N}^{e}} w^{n} v^{n, p} \leq \bar{v}^{p} \quad \forall p \in \mathcal{P}_{f}^{e}, e \in \mathcal{E}_{f}, f \in \mathcal{F}^{S t}
\end{aligned}
$$

Constraint (13) for pair $(n, p)$ defines the surplus variable $s^{n, p}$, such that the first part of the lhs of the constraint gives the expected value of function $f$ for any of the 
strategic scenarios in set $\Omega_{n}$ up to stage $e(n)$. Observe that the reason for calling it 'expected' is due to considering the set of operational scenarios (with the related weight $w^{\pi}$ for each scenario $\pi$ ) in each ancestor strategic node $n^{\prime}$ to node $n$, including itself. Remember that $\sum_{\pi \in \Pi_{n^{\prime}}} w^{\pi}=1$. Notice also that vector $b_{f}^{q}$ refers to the coefficients in period $t(q)$ for scenario $\pi$ in structure $\operatorname{graph}_{n}$. However, it simply refers to the coefficients in the reference period for scenario $\pi$ in structure $t r e e_{n}$ (and, then, those coefficients are weighted with the number of periods $\left|\mathcal{T}^{e\left(n^{\prime}\right)}\right|$ of the related stage $e\left(n^{\prime}\right)$ ). The lhs of constraints (15) and (16) give the expected surplus and expected fraction of strategic scenarios with surplus on reaching threshold $\phi^{p}$ up to stage $e$, res. The concept of the expected cost surplus (15) of the objective value on satisfying a given threshold has its roots in the Integrated Chance Constraints concept introduced in Klein Haneveld (1986), see also Klein Haneveld and Vlerk (2006). Note: Constraints (16) have their roots in the chance-constrained concept introduced in the seminal work (Charnes et al. 1958).

Following the rationale in Pflug (2000) for the CVaR measure, it can be shown that TSD is a coherent risk measure, according to the standards set up in Artzner et al. (1999), Artzner et al. (2007) for the properties, namely, translation invariance, positive homogeneity, monotonicity and convexity.

The TSD risk reduction has an interesting add-value, since it controls the periodbased peak of the values of the functions of concern in given intermediate stages, such that the longer the time horizon, the more useful the strategy is, see Alonso-Ayuso et al. (2018). However, its time inconsistency is a drawback. Notice that the outlook of the scenarios that do not belong to the group with one-to-one correspondence with a strategic node in the scenario tree (and, then, they do not occur in that situation) do have influence in the solution for that group. To prove the statement it can be observed in model TSD that the solution for node $n$, for $n \in \mathcal{N}^{e}, e \in \mathcal{E}$, is affected by the satisfaction of the risk constraint system (15)-(16) related to stage $e$ over all of its nodes in set $\mathcal{N}^{e}$, for $e \in \mathcal{E}_{f}, f \in \mathcal{F}^{S t}$. However, by construction, none of the scenarios in set $\Omega_{n^{\prime}}$ is to occur in that case, since $\Omega_{n} \cap \Omega_{n^{\prime}}=\emptyset$, for $n, n^{\prime} \in \mathcal{N}^{e}: n^{\prime} \neq n$.

\subsection{Strategic node-based time-consistent SD measure for operational functions}

The expected conditional second-order stochastic dominance (ECSD) measure that we proposed elsewhere (Escudero et al. 2017b) for operational multistage scenario tree-based problems is specialized in this section as a measure for risk reduction in the structures graph $_{n}$ and $\operatorname{tree}_{n}$, for $n \in \mathcal{N}$. Here, a set of operational functions, say $\mathcal{F}^{O p}$, and a subset of stages, say $\mathcal{E}_{f}$ for $f \in \mathcal{F}^{O p}$ are given for risk control in the sets of operational scenarios. Note: $\mathcal{F}^{S t} \cap \mathcal{F}^{O p}$ could be an empty set.

Thus, the set of profiles, denoted as $\mathcal{P}_{n}^{f}$, is associated with strategic node $n$, for $n \in \mathcal{N}^{e}, e \in \mathcal{E}_{f}, f \in \mathcal{F}^{O p}$, instead of been associated with stage $e(n)$ as it is in TSD. For each profile $p \in \mathcal{P}_{n}^{f}$, the following parameters are required:

$\gamma^{p}$, function $f$ threshold to consider in any operational node $\pi$, for $\pi \in \Pi_{n}$.

$\tilde{s}^{p}$, upper bound of the surplus on reaching threshold $\gamma^{p}$ in any operational node $\pi$, for $\pi \in \Pi_{n}$. 
$\bar{s}^{p}$, upper bound of the expected surplus on reaching threshold $\gamma^{p}$ in the set of operational nodes $\Pi_{n}$.

Let $s^{\pi, p}$ denote the continuous variable that takes the surplus of the value of function $f$ over threshold $\gamma^{p}$ in operational scenario $\pi$ for strategic node $n$.

The ECSD constraint system can be expresses as

$$
\begin{gathered}
\frac{1}{\left(1+\rho^{t(n)}\right)}\left(\left|\mathcal{T}^{e(n)}\right|_{: e(n)>\tilde{e})} \sum_{q \in \mathcal{A}_{n}^{\pi}} b_{f}^{q} z^{q}-s^{\pi, p} \leq \gamma^{p}, \quad 0 \leq s^{\pi, p} \leq \tilde{s}^{p}\right. \\
\forall \pi \in \Pi_{n}, p \in \mathcal{P}_{n}^{f}, n \in \mathcal{N}^{e}, e \in \mathcal{E}_{f}, f \in \mathcal{F}^{O p} \\
\sum_{\pi \in \Pi_{n}} w^{\pi} s^{\pi, p} \leq \bar{s}^{p} \quad \forall p \in \mathcal{P}_{n}^{f}, n \in \mathcal{N}^{e}, e \in \mathcal{E}_{f}, f \in \mathcal{F}^{O p} .
\end{gathered}
$$

Similarly to constraint (13) for TSD, constraint (17) for pair $(\pi, p)$ defines the surplus variable $s^{\pi, p}$, such that the first part of the lhs of the constraint gives the value of function $f$ that corresponds to operational scenario $\pi$ for strategic node $n$. We refer to the above remark for (13) on relation to coefficient $b_{f}^{q}$, scenario $\pi$ and the structures graph $_{n}$ and tree $_{n}$. The lhs of constraint (18) gives the expected surplus of the value of function $f$ on reaching the threshold in the set of operational scenarios of node $n$.

Let the following additional remarks:

1. The ECSD constraint system (17), (18) for structure tree $_{n}$ as a risk averse functional has certain parallelism with the risk averse functional CVaR (Gaivoronski and Pflug 2005; Rockafellar and Uryasev 2000) considered in Kaut et al. (2014), Werner et al. (2013) for multistage multiperiod stochastic problems.

2. No first-order stochastic dominance functional is considered in this type of ECSD. The main reason is that, probably, the cardinality $\left|\Pi_{n}\right|$ is not high enough. And, in any case, the related number of $0-1$ variables $\sum_{f \in \mathcal{F} o_{p}} \sum_{e \in \mathcal{E}_{f}} \sum_{n \in \mathcal{N}^{e}} \sum_{p \in \mathcal{P}_{n}^{f}}\left|\Pi_{n}\right|$ that would be required is big enough to make the model's solving unaffordable for today HW/SW standards.

3. According to the definition of the time consistency property of risk averse measures given in Homem-de-Mello and Pagnoncelli (2016), it is not difficult to prove that ECSD in tree $_{n}$ is a strategic node-based time-consistent functional. See also Kormik and Morton (2015), Pflug and Pichler (2015), Rudloff et al. (2014), Ruszczyński (2010), Shapiro (2009), Shapiro and Pichler (2016), among others.

4. A very interesting functional could be the mixture of the TSD and ECSD constraint systems in the same model, no matter the additional difficulty of the decomposition methodology that is required for problem solving. Good results are reported in Alonso-Ayuso et al. (2018) for a mixture of time-consistent and time-inconsistent versions of the CVaR functional (Gaivoronski and Pflug 2005; Pflug 2000; Rockafellar and Uryasev 2000).

So, the model that is proposed in this work can be expressed as the objective function (2) to be minimized, subject to the following constraint system: strategic node-based constraints (3)-(7), constraints for linking strategic and operational variables (8)(10), operational node-based constraints (11), (12), cross strategic node-based TSD constraints (13)-(16), and cross operational node-based ECSD constraints (17), (18). 


\section{Discussion and research plan outline}

In this work, we have presented the modeling framework for a multistage multiperiod stochastic mixed 0-1 model for capacity expansion planning (CEP) problems in a broad sense along a long-term time horizon. Two types of decisions are considered, namely strategic ones (i.e., decisions on the selection, location, capacity and timing of infrastructure elements) in a system, and operational decisions based on the available infrastructure elements in the periods of the time horizon. Those periods are partitioned in stages and, without loss of generality, the first period of each stage is chosen for the strategic decision making. Given the dynamic nature of the problem, the realization of main parameters is uncertain. Two types of uncertainties are considered, namely the strategic and operational ones. Examples of the former are the investment cost of the infrastructure elements in the system and the product or service demand intervals, among others. Examples of the latter are the elements' disruption and operational costs, and product or service specific demand, among other local parameters. A finite set of discrete scenarios, represented in a multistage scenario tree, is taken into account for considering the two types of uncertainties, contrary to the traditional approach in practice that considers expected values for the uncertain parameters.

Additionally, contrary to the traditional approach in stochastic optimization literature for dynamic CEP (as is the case) and others, the uncertain parameters in the scenario tree are not independently considered of their strategic or operational character. In accordance with that, a set of scenarios for the uncertain operational parameters are to be linked in an independent isolated way with the strategic nodes in the multistage scenario tree. The rationale behind the partition of the uncertainty's realizations in strategic and operational ones is that an operational uncertain parameter should not have any influence in future strategic decisions in an individual basis. It should just have influence in the operational decisions in its related strategic node. As a scenario set, those realizations do also influence the strategic decisions in the node and related ancestors.

The proposed modeling framework considers that the parameter uncertainty is stagewise. In fact, the probability distribution of the realization of the strategic parameters depends upon the realization of the uncertain parameters in the previous stage. A set of operational scenarios has been represented as a graph-based structure up to a given stage and as a two-stage tree structure after that stage, and it is basically problem dependent. The graph-based structure explicitly considers the modeling of the activity at the periods in the stages (by assuming that the activity in one period influences on the successors). On the other hand, the two-stage tree structure rooted with a replica of the strategic node in the multistage tree assumes that the activity in a period of a stage is independent of the activity in the other periods of the same stage. Notice that it is assumed in the two-stage tree structure the periods in a stage share the same set of available infrastructure elements.

In spite of the strategic-operational partition in the scenario tree, the model's dimensions are very high. As an illustrative example, let us consider the risk neutral (RN) version of a multistage pure $0-1$ stochastic model for a well known rapid transit network design (RTND) problem. Here, since the demand is only for passenger transport services, there is no place for operational graph structures and, thus, $\tilde{e}=0$. The 
problem has 9 station-nodes, 30 edges and 72 passenger groups, where the proof-ofconcept symmetric scenario tree consisted of 3 stages and 7 strategic nodes $(1,2$ and 4 nodes in stages 1, 2 and 3, res.) and 8 operational scenarios for each strategic node, see Figs. 1 and 4. For that case, the number of constraints and (all binary) variables in the model are 462,000+ and 291,000+, res., see Cadarso et al. (2018). Plain use of the CPLEX engine v12.6.2 for problem solving reaches the $10 \mathrm{~h}$ time limit (in a processor Intel Xeon E5-2620, 2.4Ghz, 6 cores, RAM 64GB) without proving the optimality of the incumbent solution, its optimality gap is $5.77 \%$. Let the RTND problem with a larger scenario tree (4 stages and 40 strategic nodes with 8 operational scenarios each), where the constraints and variables that force a penalization of the passenger transfer from one line to another are removed. Its dimensions are $2.4 \mathrm{M}+$ constraints and $1.6 \mathrm{M}+$ variables. For illustrative purposes, the optimization of that reduced model was stopped due to running out of memory after $85 \mathrm{~h}$ and $6 \mathrm{~min}$, as reported in Cadarso et al. (2018). In any case, that model's reduced version gives an unacceptable solution for a real-life rapid transit network operational problem, since it allows many passenger transfers on their way from origin to destination. A solution for the original model has been obtained by a fix-and-lazy scenario clustering-based matheursitic with a dynamic aggregation and de-aggregation scheme for the operational scenarios in the two-stage trees. It exploits the scenario node-based nice structure of the $\mathrm{RN}$ version of the original model. The schemes for variable fixing and cut generation and appending presented there were key ingredients for obtaining good solutions in reasonable computational time, given the very large model dimensions.

A matheuristic version of the Nested Stochastic Decomposition (NSD) methodology, in particular, the Stochastic Dynamic Programming (SDP) algorithm for stagewise dependent uncertainty is one of the most suitable methodologies for solving dynamic problems as CEP and others, see our works (Aldasoro et al. 2015; Cristobal et al. 2009; Escudero et al. 2017b). One of the main reasons for its good performance is that the partition of stages in the so-named stage blocks (of consecutive stages) makes the NSD decomposition procedure easier, where each iteration has two main steps. The first step, the so-named forward one, is devoted to improving the incumbent solution by sequentially solving each stage block related submodel. The second step, the so-named backward one, is devoted to generating and appending (hopefully) strong cuts in the stage block submodel to be used by the forward step of the next iteration. Now, the constraint system of the RN stage block submodel includes independent submodels. Each one is supported by a subtree rooted with a strategic node in the set of immediate successor nodes of each leaf strategic node in the previous stage block.

Notice that for solving real-life multistage strategic problems with operational graph and tree structures, RN-based models could provide solutions with high cost variability in the scenarios. Thus, risk averse functionals should be dealt with, so that low-probability high-negative values in those functions are prevented. The type of risk management to address in our research plan considers a mixture of the following strategic node-based time-consistent and time-inconsistent multi-function functionals for risk averse: 
1. The time stochastic dominance TSD functional on the (strategic and operationalbased) values of the chosen functions up to the nodes in modeler-driven intermediate stages along the time horizon, see Sect. 3.1. Notice that the functional destroys the nice structure of the RN model, due to the many related cross scenario node constraints that are involved.

2. The expected conditional stochastic dominance ECSD functional on the values of the operational functions at the operational scenarios in the strategic nodes, see Sect. 3.2. Notice that the functional does not destroy the structure of the RN model and, on the other hand, it strongly increase the dimensions of the strategic node-based constraint system.

However, in spite of the advantages of using the NSD methodology for dynamic problem solving, it has still some drawbacks. In fact, we have observed in our experience mainly with stochastic mixed $0-1$ models that the NSD's efficiency is reduced for those problems with stepwise dependent non-Markovian processes where some variables link a high number of consecutive stages. In any case, it is a good approach for solving very large-sized stochastic problems where the linking is only required for each pair of consecutive stage blocks. For other types of stochastic problems a good feasible solution may require a high number of NSD iterations and, additionally, the lower bound on the optimal solution that is automatically obtained could be weak for that type of problems. The main reason is the way in which the so-called Expected Future Value of the objective function is calculated in the backward steps. To avoid that significant drawback, the modeling framework that is proposed in this work allows, by construction, that the model be an easier stepwise dependent Markovian process.

Given the character of the consecutive stage blocks linking (i.e., state) variables, our NSD research would benefit from the splitting variable scheme considered in Zou et al. (2018) for state 0-1 variables. We will also consider in the NSD forward step a fix-and-lazy scenario clustering-based matheuristic for getting solutions, where the risk averse constraint systems are considered as proposed next. Notice that the TSD approach includes cross strategic node constraints in the submodels to solve at each NSD iteration. So, a scheme is required for decomposing the submodel. We are considering a scenario Cluster Dualization approach for the now required splitting constraints of the TSD variables, jointly with a Lagrangean Relaxation of the TSD constraints. So, for that purpose, we will follow in our research plan the scheme that has been presented in Escudero et al. (2017a).

Acknowledgements This research has been partially supported by the projects: MTM2015-63710 and MTM2016-79765 from the Spanish Ministry of Economy and Competitiveness. The authors like to thank the positive criticism of their colleagues Antonio Alonso-Ayuso, Luis Cadarso, F. Javier Martín-Campo and Angel Marín that helped to improve the presentation of the work.

\section{References}

Aldasoro U, Escudero LF, Merino M, Monge JF, Pérez G (2015) On parallelization of a Stochastic Dynamic Programming algorithm for solving large-scale mixed 0-1 problems under uncertainty. TOP 23:703742 
Alonso-Ayuso A, Carvallo F, Escudero LF, Guignard M, Pi J, Puranmalka R, Weintraub A (2014) On the optimization of copper extraction in mining under uncertainty in copper prices. Eur J Oper Res 233:711-726

Alonso-Ayuso A, Escudero LF, Guignard M, Weintraub A (2018) Risk management for forestry planning under uncertainty in demands and prices. Eur J Oper Res 267:1051-1074

Artzner P, Delbaen F, Eber L, Health D (1999) Coherent measures of risk. Math Finance 9:203-228

Artzner P, Delbaen F, Eber L, Health D, Ku H (2007) Coherent multi-period risk adjusted values and Bellman's principle. Ann Oper Res 152:5-22

Cadarso L, Escudero LF, Marín A (2018) On strategic multistage operational multihorizon 0-1 stochastic optimization for the Rapid Transit Network Design (RTND) problem. Eur J Oper Res. https://doi.org/ 10.1016/j.ejor.2018.05.041

Charnes A, Cooper WW, Symonds SH (1958) Cost horizons and certainty equivalents: an approach to stochastic programming of heating oil. Manag Sci 4:235-263

Cristobal MP, Escudero LF, Monge JF (2009) On Stochastic Dynamic Programming for solving large-scale tactical production planning problems. Comput Oper Res 36:2418-2428

Dentcheva D, Ruszczynski A (2003) Optimization with stochastic dominance constraints. SIAM J Optim 14:548-566

Escudero LF, Garín A, Merino M, Pérez G (2016) On time stochastic dominance induced by mixed integerlinear recourse in multistage stochastic programs. Eur J Oper Res 249:164-176

Escudero LF, Garín A, Unzueta A (2017a) Cluster Lagrangean decomposition for risk averse in multistage stochastic optimization. Comput Oper Res 85:154-171

Escudero LF, Monge JF, Romero-Morales D (2017b) On time-consistent stochastic dominance risk averse measure for Tactical Supply Chain Planning under uncertainty. Comput Oper Res. https://doi.org/10. 1016/j.cor.2017.07.011

Gaivoronski AA, Pflug G (2005) Value-at-risk in portfolio optimization: properties and computational approach. J Risk 7:11-31

Gollmer R, Gotzes U, Schultz R (2011) A note on second-order stochastic dominance constraints induced by mixed-integer linear recourse. Math Program Ser B 126:179-190

Gollmer R, Neise F, Schultz R (2008) Stochastic programs with first-order stochastic dominance constraints induced by mixed-integer linear recourse. SIAM J Optim 19:552-571

Homem-de-Mello T, Pagnoncelli BK (2016) Risk aversion in multistage stochastic programming: a modeling and algorithmic perspective. Eur J Oper Res 249:188-199

Kaut M, Midthun KT, Werner AS, Tomasgard A, Hellemo L, Fodstad M (2014) Dual-level scenario trees scenario generation and applications in energy planning. Comput Manag Sci 11:179-193

Klein Haneveld WK (1986) Duality in stochastic linear and dynamic programming. Lecture notes in economics and mathematical systems. Springer, Berlin

Klein Haneveld WK, van der Vlerk MH (2006) Integrated chance constraints: reduced forms and an algorithm. Comput Manag Sci 3:245269

Kormik V, Morton DP (2015) Evaluating policies in risk avrse multistage stochastic programming. Math Program Ser A 152:275-300

Pflug GCh (2000) Some remarks on the value-at-risk and the conditional value-at-risk. In: Uryasev S (ed) Probabilistic constrained optimization. Methodology and applications. Springer, Berlin, pp 272-281

Pflug GCh, Pichler A (2015) Time consistent decisions and temporal decomposition of coherent risk functional. Math Oper Res 41:682-699

Pflug G Ch, Römisch W (2007) Modeling, measuring and managing risk. World Scientific, Singapore

Rockafellar RT, Uryasev S (2000) Optimization on conditional value-at-risk. J Risk 2:21-41

Rudloff B, Street A, Valladão DM (2014) Time consistency and risk averse dynamic decision models: definition, interpretation and practical consequences. Eur J Oper Res 234:743-750

Ruszczyński A (2010) Risk-averse dynamic programming for Markov decision processes. Math Program 125:235-261

Shapiro A (2009) On a time consistency concept in risk averse multistage stochastic programming. Oper Res Lett 37:143-147

Shapiro A, Pichler A (2016) Time and dynamic consistency of risk averse stochastic programs. Optimization online http://www.optimization-online.org/DBHTML/2016/09/5654. Accessed 24 May 2018

Vespucci MT, Bertocchi M, Zigrino S, Escudero LF (2013) Stochastic optimization models for the power generation capacity expansion problem with risk management. In: 10th International conference IEEE on the European energy market EEM13, Stockholm. https://doi.org/10.1109/EEM.2013.6607352 
Werner AS, Pichler A, Midthun KT, Hellemo L, Tomasgard A (2013) Risk measures in multihorizon scenarios tree. In: Kovacevic R, Pflug GCh, Vespucci MT (eds) Handbook of risk management in energy production and trading. Springer, Berlin, pp 177-201

Zou J, Ahmed S, Sun XA (2018) Sochatic dual dynamic intger programming. Math Program. https://doi. org/10.1007/s10107-018-1249-5 